\title{
Influence of Peritoneal Dialysis and Hemodialysis on Blood Lipids of Uremic Patients
}

\author{
Ying Shi \\ The Third Affiliated Hospital of Qiqihar Medical University,161000, China \\ email: shiying568@126.com
}

Keywords: Uremia; Blood lipid; Hemodialysis; Peritoneal dialysis

\begin{abstract}
Objective: To investigate the influence of peritoneal dialysis and hemodialysis on blood lipids of uremic patients; Methods: 100 uremic patients admitted to the hospital were selected as observation objects, clinical data of patients were collected and analyzed retrospectively. 50 patients received peritoneal dialysis treatment, 50 patients underwent hemodialysis treatment. Change conditions of metabolism for patients in the two groups before and after treatment were observed and compared. Results: The serum total cholesterol $(6.46 \pm 0.91 \mathrm{mmol} / \mathrm{L})$, triglycerides $(2.36 \pm 0.65$ $\mathrm{mmol} / \mathrm{L})$, low-density lipoprotein $(3.75 \pm 0.68 \mathrm{mmol} / \mathrm{L})$ and apolipoprotein $\mathrm{B}(1.19 \pm 0.15 \mathrm{mmol} / \mathrm{L})$ of peritoneal dialysis patients after treatment were significantly higher than the values before treatment $(\mathrm{P}<0.05)$. The high-density lipoprotein (HDL) and apolipoprotein A (A poA) of peritoneal dialysis patients after treatment were significantly lower than values before treatment $(\mathrm{P}<0.05)$. Various lipid metabolism indicator comparison $\mathrm{P}>0.05$ of hemodialysis patients before and after treatment was not statistically significant. Conclusion: Peritoneal dialysis has more prominent influence on blood lipids of uremic patients compared with hemodialysis. Doctors should pay attention to lipid-lowering therapy in uremic patients.
\end{abstract}

\section{Introduction}

Uremia mainly refers to end stage of patient renal insufficiency, wherein complicated cardiovascular disease is significant cause of death in patients with uremia. However, hyperlipidemia is risky factor for cardiovascular disease[1-2]. Studies have shown that dialysis treatment is mostly adopted for clinical treatment of uremia. It has better effect on maintaining patient physiological indicators stable. Patient survival rate can be prominently improved through improving blood lipid abnormalities in patients with uremia. However, blood lipid level of uremia after dialysis treatment is not surely reported currently[3]. Therefore, 100 uremic patients admitted by our hospital from July 2012 to October 2013 were regarded as study objects in the paper. Blood lipid change conditions before and after dialysis and hemodialysis treatment are observed and discussed, and the conditions are reported below.

\section{Materials and Methods}

General Information. Our hospital accepted 100 uremic patients from July 2012 to October 2013, wherein 50 patients accepted peritoneal dialysis treatment, including 36 male patients and 14 female patients. The patient age was from 42 to 61 years with mean age of $(52.3 \pm 9.0)$. Treatment duration of patients lasted for 0.5 to 15 years with average time of $(4.6 \pm 1.0)$ years; 50 patients accepted hemodialysis treatment, including 32 male patients and 18 female patients. Patient age was from 42 to 64 years with mean age of $(49.5 \pm 8.5)$. Treatment duration of patients lasted for 0.7 to 15 years with average time of ( $4.4 \pm 0.9$ years). Patients in the group did not receive blood lipid lowering treatment by any drug within 90 days before the treatment. Patient eGFR was $15 \mathrm{ml} / \mathrm{min}$ or less. Patients with primary hyperlipidemia, diabetic nephropathy and severe liver disease were excluded. Age, treatment time, etc. of patients in each group were compared, $\mathrm{P}>0.05$, and the results were not statistically significant and comparable. 
Methods. (A)Peritoneal Dialysis Treatment Methods 50 uremic patients underwent peritoneal dialysis, dialysate lactate of duplex system was adopted for hemodialysis, wherein daily dialysate exchange of 33 patients was $6 \mathrm{~L}$, and daily dialysate exchange of 17 patients was $8 \mathrm{~L}$.

(B)Hemodialysis Treatment . 50 patients underwent hemodialysis treatment, dialysis machine was adopted for hemodialysis. Double cellulose acetate membrane and bicarbonate dialysate were used. Edge anticoagulant heparin was applied, dose of unfractionated heparin anticoagulant was $1 \mathrm{mg} / \mathrm{kg}$ to $6 \mathrm{mg} / \mathrm{kg}$, or using low molecular weight heparin was adopted. When edge heparin anticoagulation dialysis was conducted, blood flow rate should be $200 \mathrm{ml} / \mathrm{min}$ to $280 \mathrm{ml} / \mathrm{min}$, dialysate flow rate should be $500 \mathrm{ml} / \mathrm{min}$, patient hemodialysis treatment time was $4.5 \mathrm{~h}$ each time, and the patient should accept dialysis treatment 2 to 3 times each week.

(C)Symptomatic and Supportive Treatment Method. Patients with uremia in the group accepted high-quality low -protein diet completely and protein according to dose of $0.6 \mathrm{~g} / \mathrm{kg}$ once a day. Coated aldehyde oxidized starch was given by oral administration. Dosage was $5 \mathrm{~g}$ each time 3 times a day. The patients should underwent water, acid-base balance and other symptomatic treatments[4].

(D)Blood Lipid Determination Method . Blood lipids of patients after treatment for six months was measured. Measurement methods: the patients were fasted for $10 \mathrm{~h}$, fasting blood in the morning was extracted, arm venous blood was collected for serum separation. Blood lipid should be determined in the same day, and Automatic biochemical analyzer was adopted for testing.

Indicator Observation. Blood lipid metabolism change conditions of patients in all groups before and after treatment were observed, including serum total cholesterol (TCH) and triglyceride (TG). The two indictors were tested by enzymic method; high-density lipoprotein (HDL) and low-density lipoprotein protein (LDL) were tested by masked direct detection assay, apolipoprotein A (A poA) and apolipoprotein B (A poB) were tested by immune nephelometry.

Statistical Methods. Data were analyzed by SPSS17.0 and t test measurement data (mean \pm standard deviation), data was compared $\mathrm{P}<0.05$, and the difference was statistically significant.

\section{Results}

Blood Lipid Comparison of Peritoneal Dialysis Patients Before and After Treatment. Various blood lipid testing indicators of peritoneal dialysis patients after treatment were prominently different compared with that before treatment, wherein test levels of TCH, TG, LDL and A poB were significantly increased, test levels of HDL and A poA were prominently lowered, blood lipid comparison among groups $\mathrm{P}<0.05$ was statistically significant, and specific conditions are shown in Table 1.

Table 1 Blood Lipid Determination Comparison Condition of Peritoneal Dialysis Patients before and after Treatment

\begin{tabular}{ccccccc}
\hline Time & $\begin{array}{c}\text { TCH } \\
(\mathrm{mmol} / \mathrm{L})\end{array}$ & $\begin{array}{c}\text { TG } \\
(\mathrm{mmol} / \mathrm{L})\end{array}$ & $\begin{array}{c}\mathrm{HDL} \\
(\mathrm{mmol} / \mathrm{L})\end{array}$ & $\begin{array}{c}\mathrm{LDL} \\
(\mathrm{mmol} / \mathrm{L})\end{array}$ & $\begin{array}{c}\text { A poB } \\
(\mathrm{mmol} / \mathrm{L})\end{array}$ & $\begin{array}{c}\text { A poA } \\
(\mathrm{mmol} / \mathrm{L})\end{array}$ \\
\hline Before treatment (50 cases) & $5.93 \pm 0.61$ & $1.74 \pm 0.53$ & $0.79 \pm 0.26$ & $3.22 \pm 0.63$ & $0.83 \pm 0.41$ & $0.89 \pm 0.19$ \\
After treatmen (50 cases) & $6.46 \pm 0.91$ & $2.36 \pm 0.65$ & $0.61 \pm 0.28$ & $3.75 \pm 0.68$ & $1.19 \pm 0.15$ & $0.63 \pm 0.20$ \\
$\mathrm{t}$ & 3.421 & 5.227 & 3.331 & 4.043 & 5.831 & 6.664 \\
$\mathrm{P}$ & 0.001 & 0.000 & 0.001 & 0.000 & 0.000 & 0.000 \\
\hline
\end{tabular}

Blood Lipid Comparison Condition of Hemodialysis Patients before and after Treatment. Various blood lipid test indicators of uremic patients accepting hemodialysis treatment were not prominently changed before and after treatment. Blood lipid comparison among groups $\mathrm{P}>0.05$ was not statistically significant, and specific conditions were shown in Table 2. 
Table 2 Blood Lipid Determination Comparison Condition of Hemodialysis Patients before and after Treatment

\begin{tabular}{ccccccc}
\hline Time & TCH(mmol/L) & $\begin{array}{c}\text { TG } \\
(\mathrm{mmol} / \mathrm{L})\end{array}$ & $\begin{array}{c}\mathrm{HDL} \\
(\mathrm{mmol} / \mathrm{L})\end{array}$ & $\begin{array}{c}\mathrm{LDL} \\
(\mathrm{mmol} / \mathrm{L})\end{array}$ & $\begin{array}{c}\text { A poB } \\
(\mathrm{mmol} / \mathrm{L})\end{array}$ & $\begin{array}{c}\text { A poA } \\
(\mathrm{mmol} / \mathrm{L})\end{array}$ \\
\hline Before treatment(50 cases) & $5.67 \pm 0.76$ & $1.83 \pm 0.51$ & $0.79 \pm 0.29$ & $3.26 \pm 0.68$ & $0.91 \pm 0.23$ & $1.22 \pm 0.22$ \\
After treatmen (50 cases) & $5.82 \pm 0.78$ & $1.86 \pm 0.48$ & $0.73 \pm 0.28$ & $3.27 \pm 0.66$ & $0.96 \pm 0.11$ & $1.26 \pm 0.20$ \\
$\mathrm{t}$ & 0.974 & 0.303 & 1.052 & 0.075 & 1.387 & 0.951 \\
$\mathrm{P}$ & 0.333 & 0.763 & 0.295 & 0.941 & 0.169 & 0.344 \\
\hline
\end{tabular}

Blood Lipid Comparison of Peritoneal Dialysis Patients and Hemodialysis Patients After Treatment. Four blood lipid determination indicators of TCH, TG, LDL and A poB of peritoneal dialysis patients after treatment were prominently higher than the determination level of hemodialysis patients after treatment. Two blood lipid determination indicators of HDL and A poA of peritoneal dialysis patients after treatment were prominently lower than the determination level of hemodialysis patients after treatment, and blood lipid comparison among groups $\mathrm{P}<0.05$ was statistically significant, and specific statistical data was shown in Table 3.

Table 3 Blood Lipid Determination Comparison Statistical Data of Peritoneal Dialysis and Hemodialysis Patients after Treatment

\begin{tabular}{ccccccc}
\hline Category & $\begin{array}{c}\mathrm{TCH}(\mathrm{mmol} / \\
\mathrm{L})\end{array}$ & $\mathrm{TG}(\mathrm{mmol} / \mathrm{L})$ & $\begin{array}{c}\mathrm{HDL} \\
(\mathrm{mmol} / \mathrm{L})\end{array}$ & $\begin{array}{c}\mathrm{LDL} \\
(\mathrm{mmol} / \mathrm{L})\end{array}$ & $\begin{array}{c}\mathrm{A} \mathrm{poB} \\
(\mathrm{mmol} / \mathrm{L})\end{array}$ & $\begin{array}{c}\mathrm{A} \mathrm{poA} \\
(\mathrm{mmol} / \mathrm{L})\end{array}$ \\
\hline $\mathrm{t}$ & 3.776 & 4.376 & 2.143 & 3.582 & 2.165 & 15.75 \\
$\mathrm{P}$ & 0.000 & 0.000 & 0.035 & 0.001 & 0.033 & 0.000 \\
\hline
\end{tabular}

\section{Discussion}

Related Overview. Uremia mainly refers that acute renal insufficiency or chronic renal insufficiency is developed to severe stage, namely renal insufficiency is developed to end stage, endocrine dysfunction is caused by own protein metabolite accumulation, urea accumulation, electrolytes and acid-base balance disorders, etc. in patient body due to renal failure. In addition, a series of self -poisoning symptoms are caused by endocrine dysfunction. In addition, a variety of chronic kidney diseases can lead to lipid metabolism, which is manifested as hyperlipidemia. The main reasons include chylomicrons, VLDL, LDL clear-down, low-density lipoprotein synthesis, etc. Lipid substances are adhered to glomerular endothelial cells of patients. Meanwhile, the substances penetrate to subendothelial space, thereby increasing phagocytic mononuclear cells, forming foam cells, and releasing cytokines. With increase of matrix synthesis and secretion outside stimuli cells and mesangial cell proliferation, few glomerulus suffers from sclerosis, thereby leading to kidney disease aggravation in patients, and accelerating impaired renal function, and it is important factor leading to vascular complications of kidney diseases.

Result Analysis. Research shows that the majority of uremic patients are accompanied by multiple organ damage. Most patients also have metabolic disorders and other problems, wherein dyslipidemia is a more common performance. Dyslipidemia is a significant risky factor for cardiovascular disease in uremia, thereby cardiovascular disease is the main reason resulting in death of uremic patients. Furthermore, lipid metabolism should be emphasized in the clinical treatment process of uremic patients, and the symptom should be positively corrected for improving patient survival rate[5].

Dialysis treatment mainly refers that solute moves through semipermeable membrane from solution with high concentration to low concentration direction. Wherein, hemodialysis mainly refers that a part of waste contained in patient blood can be removed through dialysis equipment, namely waste can be removed through semipermeable membrane. Hemodialysis has high security and easy operation, which has been widely used in blood purification. The study results of the group show that TCH, TG, DL, A poB, HDL and A poA testing levels of hemodialysis patients after treatment for six months were prominently lowered, blood lipid testing indicators are compared with that before treatment without o significant difference $(\mathrm{P}<0.05)$. Tip: hemodialysis treatment of uremic patients has no prominent influence on blood lipid level with higher safety. The results are consistent with report. Analysis shows that the treatment method is related with hemodialysis treatment principle, etc. It includes movement of solute and water, namely blood and dialysate are arranged in artificial kidney (dialyzer), they conduct substance exchange with concentration gradient through contact by 
semipermeable membrane, thereby a part of metabolic waste contained in patient blood and excessive electrolyte in blood can move to dialysate. Components in dialysate can move to blood, including calcium ions, base, etc. low molecular weight heparin is used for maintenance, erythropoietin is promoted during hemodialysis treatment process. ACEI class antihypertensive drugs and other drugs are selected, which are beneficial to lipid metabolism of patients. In addition, dialyzers used for hemodialysis is polysulfone membrane dialyzer, which has good biocompatibility and can effectively promote improvement of blood lipid metabolism.

Dialysate in patient abdominal cavity is frequently replaced as main means for peritoneal dialysis, thereby eliminating metabolites, toxic substances, etc. in patient body. Meanwhile, the treatment method can reach the effect of correcting water and electrolyte balance disorders and so on. It regards peritoneum as a semi-permeable membrane and uses effect of gravity for infusing dialysate into peritoneal cavity of patients. Namely, formulated dialysate is infused through pipe. Then, there will be difference in solute concentration gradient on both sides of patient peritoneum, thereby moving solute on the side with high concentration to the side with low concentration. Water in low penetration side can be moved to high penetration side. Results of the group show that TG, TCH, LDL and A poB of uremic patients were prominently and effectively increased after peritoneal dialysis treatment compared with that before treatment $\mathrm{P}<0.05$ ), and HDL and A poA of patients after treatment were prominently lower than the measured level before treatment $(\mathrm{P}<0.05), \mathrm{TCH}, \mathrm{TG}, \mathrm{LDL}$ and $\mathrm{A}$ poB of peritoneal dialysis patients after treatment were prominently higher than the measured level of hemodialysis patients after treatment $(\mathrm{P}<0.05)$. Tip: peritoneal dialysis has greater influence on lipid metabolism of uremic patients. The results are consistent with the report results. Analysis believes that glucose uptake in patient peritoneal dialysis dialysate is prominently increased in the peritoneal dialysis process, thereby promoting abnormal lipid metabolism of patient with relevance. Protein loss of uremic patients after peritoneal dialysis treatment was increased, lipid metabolism was aggravated, results of the group also show that important lipase activity obstacles are main factors leading to lipid metabolism disorder of uremic patients after peritoneal dialysis, such as lipolysis lipoprotein lipase (LPL) activity decrease, decrease of liver triglyceride lipase (HTGL) and so on. In addition, fatty acid synthase activity is increased, ATP lyase activity is increased, and gene expression is enhanced, and they are also closely related to lipid metabolism in peritoneal dialysis patients. The probability of hyperlipidemia in patients will be increased during long-term peritoneal dialysis. In addition, atherosclerosis also can be triggered due to increase of LDL, TG, etc. of patient serum, and insulin resistance is also an important reason leading to high TG hyperlipidemia.

\section{Summary}

Safety of hemodialysis treatment for uremic patients is higher than that of peritoneal dialysis. Hemodialysis treatment has no significant influence on patient blood lipid. Uremic patients accepting dialysis treatment especially peritoneal dialysis treatment should positively control blood lipid and improve lipid metabolism so that residual renal function in patients can be maximally protected, thereby effectively reducing incidence of cardiovascular complications for patients and improving survival rate of patients with uremia with important significance.

\section{References}

[1] ZHOU Yan, J1 Daxi. Combination of peritoneal dialysis and hemodialysis as a new modality of treatment for end-stage renal disease. Chinese Journal of Nephrology, Dialysis \& Transplantation. 2013, 22(2):175-179.

[2] LIN Mingzeng CHEN Xiaofeng FAN Jiani, et al. Clinical study on calcium-phosphorous metabolism in uremic patients on peritoneal dialysis or hemodialysis. Chinese Journal of Blood Purification. 2013, 12(9):487-490. 
[3] YU Yusheng, ZHANG Jong, YUAN Zenghui, et al. Rate of declining residual renal function in patients on peritoneal dialysis. Chinese Journal of Nephrology, Dialysis \& Transplantation.. 2006, 15(4):340-344.

[4] JIN Donghua, SHI Yongxing, SHEN Huaying, et al. The effect of different dialysis modalities on pruritus in patients with uremia. Chinese Journal of Emergency Medicine. 2013, 22(10): 1161-1166.

[5] MO Guohua, WEI Li-ling, LI Jia-lian, et al. Effect evaluation of nursing for senile uremia patients during induction period of hemodialysis. Chinese Journal of Practical Nursing. 2012, 28(10):42-43. 